\title{
A ENFERMAGEM E A PESQUISA NO BRASIL
}

\author{
Tamara Iwanow Cianciarullo* \\ Sonia Della Torre Salzano**
}

CIANCIARULLO, T.I.; SALZANO, S.D.T. A enfermagem e a pesquisa no Brasil. Rev. Esc. Enf. USP, v. 25, n. 2, p. 195-215, ago. 1991.

A enfermagem brasileira em relação à pesquisa apresenta uma evolução acentuada nos últimos anos, com um saldo quantitativo na última década.

Os pesquisadores brasileiros localizam-se na sua maioria na categoria docente e na regiäo sudeste do Brasil.

Existem oito Centros universitários de estudos em rivel de pós-graduação, com inúmeras áreas de concentração nos cursos de mestrado.

Os cursos de doutorado localizam-se todos na região sudeste.

Existem recursos disponiveis em agências financiadoras de pesquisas para a enfermagem, porém a demanda ainda é incipiente.

A Associação Brasileira de Enfermagem tem procurado implementar medidas que visam o deservolvimento das pesquisas na área da enfermagem.

UNITERMOS: Pesquisa em enfermagem. Enfermagem - Brasil.

\section{INTRODUÇÃO}

A enfermagem brasileira vem sofrendo importantes transformações nos últimos 20 anos. Estas transformações se referem ao perfil de atuação, desempenho, territorialidade e inserção em equipes multiprofissionais, que grupos distintos de enfermeiras, mas ainda não articulados como um todo, vêm conseguindo delinear no âmbito da assistência à saúde da população.

A inserção da enfermeira brasileira num contexto assertivo, buscando o seu espaço, caracteriza-se pelas suas atividades com tendências independentes, tais como: utilização de processo de enfermagem, atuação de clínicas irdependentes, implantação de microempresas e consultoria à órgãos privados e governamentais em âmbito nacional e inter-

- Enfermeira. Professor Titular, Vice-Diretora da Escola de Enfermagem da Universidade de săo Paulo e Presidente da Comissăo de Pesquisa.

* Enfermeira. Professor Titular, Diretora da Escola de Enfermagem da Universidade de Sá Paulò. 
nacional, além da ocupação de cargos de elevado nivel como secretarias de saúde de municipios e de Estados.

Com as mudanças significativas na atuação própria das enfermeiras, o reflexo se permeia na área da pesquisa, pois, de participantes e executores de coletas de dados em épocas remotas, são as enfermeiras brasileiras hoje, as planejadoras e executoras de pequenos, médios e grandes projetos na área da assistência à saúde.

Somos atualmente 46.518 enfermeiras graduadas no país, trabalhando com 29.083 técnicos de enfermagem e 113.148 auxiliares de enfermagem. Estes números, representam ainda um subdimensionamento das necessidades do pais, mas já definem em termos de qualidade, os caminhos a percorrer para atender a demanda de serviços da coletividade brasileira, caracterizado em cerca de 150.000 .000 de habitantes.

Sabemos quantos somos, quem somos e para onde precisamos ir, porém a instrumentação deste caminho nos será fornecida, sobretudo, pelas pesquisas em desenvolvimento e a serem desenvolvidas na área, na próxima década.

\section{HISTÓRIA DA PESQUISA EM ENFERMAGEM}

A pesquisa no Brasil tem meros de oitenta anos de existência, tendo se iniciado no começo do século na busca de soluções para o problema da peste no Rio de Janeiro ${ }^{13}$.

$\mathrm{Na}$ enfermagem o primeiro trabalho documentado de investigação, foi o "Censo de 1950", caracterizando o início da investigação na área ${ }^{15}$.

A questão a ser acentuada a partir deste cocumento, refere-se à quantidade e tipologia das pesquisas em enfermagem realizadas no Brasil, no período de 1950 a 1989.

Os dados quantitativos apresentados neste trabalho são fundamentados na pesquisa realizada em 1982, apresentado no II Seminário Nacional sobre Ensino de Pós-Graduação e Pesquisa em Enfermagem, onde é analisada a produção de trabalhos de pesquisa entre os anos de 1950 e 1981. Neste estudo, foram classificados os trabalhos de investigação realizados por enfermeiros ou por outros profissionais sobre a Enfermagem ${ }^{15}$.

As fontes de referência utilizadas pela autora, corresponderam aos trabalhos computados no período de 1950 a 1981, nos seguintes periódicos e publicações: Anais de Enfermagem (1946-1954); Revista Brasileira de Enfermagem (1955-1989); Revista da Escola de Enfermagem da USP (1967-1989); Enfoque (1972-1989); Enfermagem em Novas Dimensões (1975-1979); Revista Gaúcha de Enfermagem (1976-1989); Enfermagem Atual (1978-1982); Revista Paulista de Enfermagem (1981-1989); Revista Bahiana de Enfermagem (1981-1989); Enfermagem Moderna (1983-1985); Acta Paulista (1988-1989); Dados do Cen- 
tro de Pesquisa em Enfermagem (CEPEn) da Associação Brasileira de Enfermagem (ABEn); Documentário sobre a Associação Brasileira de Enfermagem (ABEn); Banco de Teses.

As fontes de referência utilizadas para complementação do periodo estudado (1980-1989), encontram-se destacadas com asterisco e definem um subdimensionamento dos resultados, visto que nesta fase não foram computados os trabalhos do Banco de Teses. Foram incluídos nesta segunda fase (1980-1989), os volumes Informações sobre Pesquisas e Pesquisadores em Enfermagem do CEPEn e as seguintes revistas: Acta Paulista (1983-1985); Revista Bahiana de Enfermagem (19811989) "; Enfermagem Moderna (1983-1985).

Foi mantida a terminologia original para a classificação dos trabalhos, apesar de hoje, definidas as prioridades, tenhamos uma nova classificação (Anexo I) utilizada pelos órgãos financiadores e pelos pesquisadores para a classificação de pesquisas na área de Enfermagem. seguir:

Os critérios de classificação foram mantidos e são especificados a

Necessidades do Paciente: pesquisas referentes à identificação de conhecimentos, atitudes, comportamentos e necessidades sentidas em relação à saúde e assistência de enfermagem prestada a grupos de pacientes e à comunidade;

Avaliação da Assistência: pesquisas cujo objetivo foi identificar ou avaliar a problemática da assistência à saúde ou assistência de enfermagem que estava sendo prestada a indivíduos, família e comunidade;

Necessidades do Pessoal de Enfermagem: pesquisas referentes à identificação de conhecimentos, atitudes, comportamentos e necessidades sentidas, do pessoal de enfermagem (profissional e auxiliar), em relação ao seu trabalho;

Metodologia de Enfermagem: pesquisas, geralmente experimentais, que propunham métodos, técnicas, subsídios ou enfoques diferentes a serem dados à assistência de enfermagem a grupos de pacientes, famílias e comunidade e pesquisas bibliográficas ou monografias, com as mesmas finalidades;

Profissão de Enfermagem: trabalhos de pesquisa sobre o estudo de papéis, funçōes e atribuições da enfermagem na equipe de saúde, na equipe de enfermagem e nos serviços de saúde, bem como a identificação de problemas relativos à ética e ao exercício profissional;

Ensino de Enfermagem: pesquisas para identificar problemas ou propor novos métodos e técnicas de ensino de enfermagem aos niveis de graduação, pós-graduação e de formação de pessoal auxiliar e de educação continuada;

- excetuando-se o ano de 1988. 
Epidemiológicas $e$ Biológicas: pesquisas que englobaram o conhecimento e a descrição de eventos biológicos ou de agravos à saúde em grupos de indivíduos.

Os critérios para a seleção dos trabalhos analisados foram também os mesmos: - terem sido publicados em território nacional; - terem sido realizados por enfermeiros ou por outros profissionais, mas cujo tema se refere à enfermagem ${ }^{15}$.

\section{RESULTADOS E COMENTARIOS}

\section{Periodo 1950 - 1959}

Nos anos cinqüenta foram desenvolvidos dez trabalhos de investigação sobre temas que ainda hoje são relevantes para os enfermeiros brasileiros, marcando assim os primórdios da investigação em enfermagem no Brasil ${ }^{15}$.

Este periodo compreende um movimento da ABEn e das Escolas de Enfermagem, visando uma mudança curricular, a aprovação do Código de Ética Profissional e uma pesquisa realizada pela ABEn sobre Recursos Humaros e Necessidades da Enfermagem no Brasil ${ }^{21}$.

A produção de pesquisas apresenta-se em seu inicio e abrange especificamente o ensino e a profissão, refletindo a preocupação da década na busca de um espaço universitário 17,21.

Neste período percebe-se a nítida preocupação das enfermeiras com a área assistencial e com a área da educação, confirmada pela realização de dois seminários: I Seminário de Ensino de Enfermagem em 1956 e Seminário Didático Internacional sobre Levantamento em Enfermagem em $1958^{25}$, configurando o perfil das publicações em geral da Enfermagem na década de cinquienta.

\section{Periodo 1960 - 1969}

A partir de 1961 passou a ser exigido o curso colegial para o ingresso nos cursos de Enfermagem e definido currículo mínimo do curso de Graduação em Enfermagem, com duração mínima de 3 anos e 1 ano de especialização.

$\mathrm{Na}$ década de sessenta com a inserção dos cursos de Enfermagem em nivel superior e que tinham por objetivo a pesquisa e o desenvolvimento das ciências, os enfermeiros começam a preocupar-se mais com a sua própria produção de pesquisas, o que pode ser visto pelos dados da Tabela 1. Insere-se nesta década a primeira tese, elaborada por enfermeira, para o concurso de Professor Catedrático, versando sobre a profissão. A eminente Professora pertencia ao quadro de docentes da Universidade de São Paulo.

A ênfase no ensino de Enfermagem reflete-se pela realização de Seminários e Congressos sobre Didática e Pedagogia Aplicada à Enfer- 
magem $(1960,1964,1968$ e 1970), mas não aparece refletida na produção de pesquisas, visto que ocorre uma diminuição de pesquisas e de trabalhos apresentados nos Congressos Brasileiros de Enfermagem sobre ensino ${ }^{15,21}$.

Em 1964, o Congresso Brasileiro de Enfermagem inclui uma nova temática "Enfermagem e Pesquisa", repetindo-se em 196725.

Mantém-se, nesta década, o percentual de trabalhos da área assistencial e percebe-se uma acentuada produção de pesquisas visando o estudo da profissão, reflexo da alteração no sistemo. educacional na área e o surgimento de uma nova categoria profissional o Técnico de Enfermagem, em 1966.

Padilha, et al (1983) em seu estudo notaram uma ligeira elevaçāo no número de trabalhos publicados na área de Enfermagem Médico-Cirúrgica relacionados à educação e assistência, com uma acentuada diminuição de trabalhos com enfoque em procedimentos.

Observa-se assim, nesta década, um crescimento da produção científica e de trabalhos de pesquitsa em Enfermagem.

\section{Periodo 1970 - 1979}

Movimentos buscando a alteração do currículo mínimo, introduzindo as habilitações, caracterizam o início da década de setenta.

A influência do modêlo médico permeia os programas da educação formal do enfermeiro com ênfase na assistência hospitalar e domínio de técnicas. A preocupação com os recursos humanos na área da enfermagem também aparece explicitada, com a publicação de 22 trabalhos.

Com a criação de cursos de Pós-Graduação "sensu strictu" nesta década, houve um acentuado desenvolvimento da enfermagem, refletida na quantidade de pesquisas realizadas e publicadas.

A quantidade de estudos referentes aos processos assistenciais, necessidades do paciente e avaliação da assistência, representam a preocupação dos enfermeiros nesta década, onde o processo preconizado por Wanda de Aguiar Horta passa a ser divulgado na sua revista "Enfermagem em Novas Dimensões", criada em 1975 e retirada de circulação em 1979 por problemas na editoração.

Ainda neste periodo, são colocados no mercado mais quatro revistas: Enfoque (1972), Revista Gaúcha de Enfermagem (1976), Anais dos Congressos Brasileiros (1977) e Enfermagem Atual (1978), propiciando melhores condições de divulgação dos trabalhos realizados por enfermeiros.

$\mathrm{Na}$ área de Enfermagem Médico-Cirúrgica, triplica o número de publicações de trabalhos com grande ênfase na área assistencial $(81,8 \%)$, à semelhança das décadas anteriores, não sendo possível diferenciar aqui o desenvolvimento e a aplicação do processo de enfermagem dado o método de classificação utilizado pelos autores ${ }^{17}$. 
Vieira (1980) afirma que além dos cursos de Pós-Graduação, com seus respectivos trabalhos de conclusão, a obrigatoriedade de qualifica. ção do corpo docente, principalmente nas Escolas vinculadas às Universidades, onde a progressão na carreira é obrigatória, vem reforçar a produção científica dos enfermeiros brasileiros.

A evolução quantitativa da produção de pesquisas (320) representa um salto na área de investigação em enfermagem nesta década. (Tabela 2)

\section{Período $1980-1989$}

O surgimento de novas propostas de reformas de organização de serviços de saúde e de estratégias de ações integradas, definiram a década de oitenta em termos de contexto, caracterizando os caminhos da enfermagem nas propostas de atenção primária na saúde.

Cabe destacar que nesta década, foram incluídas no mercado editorial de enfermagem, quatro novas revistas: Revista Paulista de Enfermagem, Acta Paulista, Revista Bahiana de Enfermagem e Enfermagem Moderna.

O crescimento da produção de pesquisas aparece claramente em todas as áreas, mantendo-se as mesmas proporções encontradas na década anterior. (Tabela 1)

Ao estudarmos a classificação das pesquisas em relação às décadas de cada uma das áreas, podemos perceber que no mínimo $70 \%$ das pesquisas publicadas no Brasil, localizam-se nesta década chegando a $82,4 \%$ na área de necessidades do paciente. (Tabela 2)

Percebe-se ainda, que o enfoque central das atenções das enfermeiras, vincula-se à assistência, visto que os trabalhos referem-se em maior percentual a Necessidades do Paciente $(82,4 \%)$, Avaliação de Assisténcia $(77,7 \%)$ e Metodologia de Enfermagem $(75,3 \%)$. (Tabela 2)

Em relação às áreas específicas de enfermagem, alguns trabalhos permitem confirmar estes resultados em relação à centralização de pesquisas na assistência da enfermagem 4,5,9,10,14,16,22.

Visualizando o conjunto em sua evolução temporal, podemos afirmar que em todas as áreas, houve um crescimento acentuado da produção de pesquisas, definindo-se a última década com uma surpreendente produção de pesquisas em Enfermagem.

\section{Perfil dos Pesquisadores}

Nas décadas de 50 e 60 , os enfermeiros não publicaram pesquisas de uma maneira sistematizada. Todavia, nos campos de trabalho intra e extra mural, muitos destes enfermeiros contribuíram com o saber específico de outras áreas como por exemplo o da medicina. 


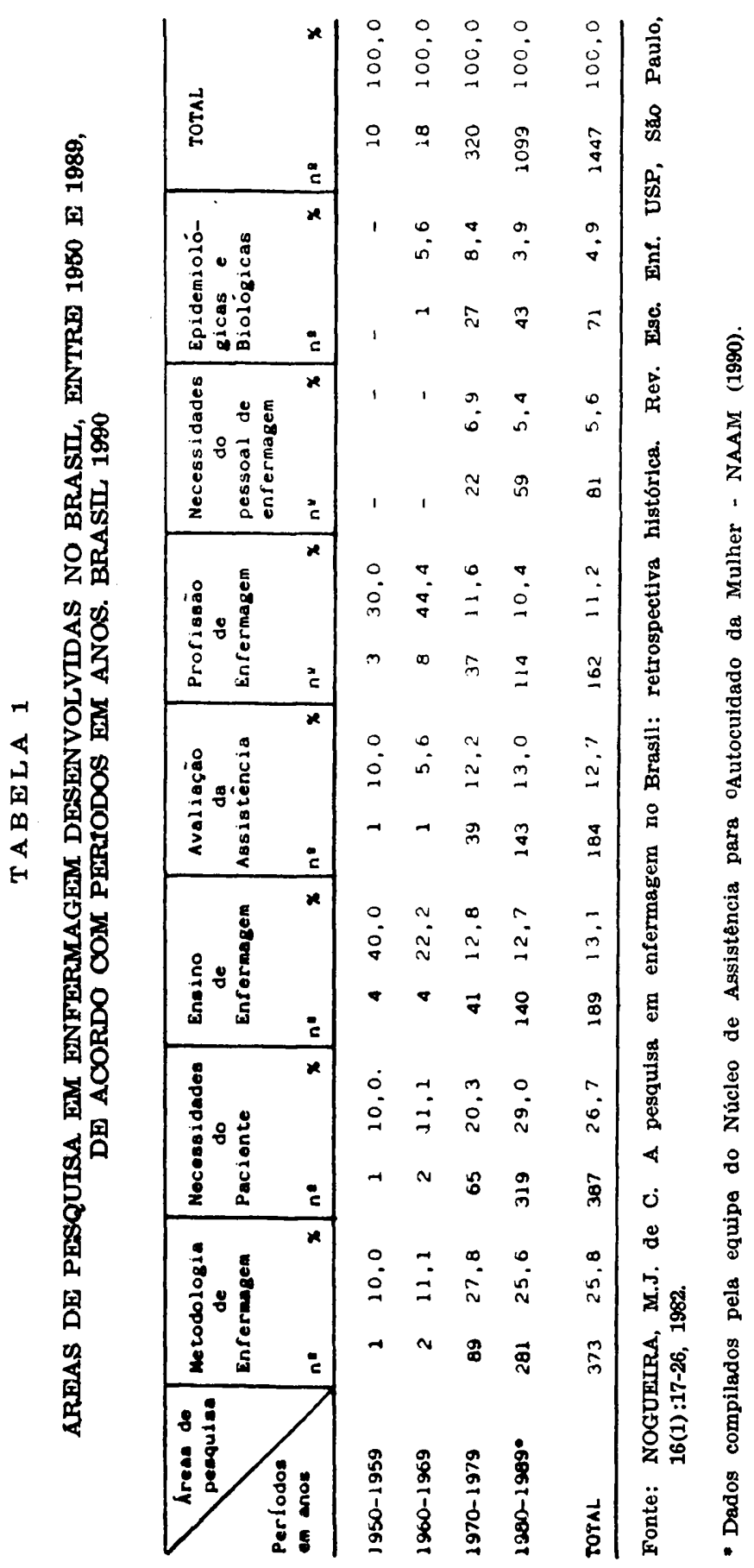




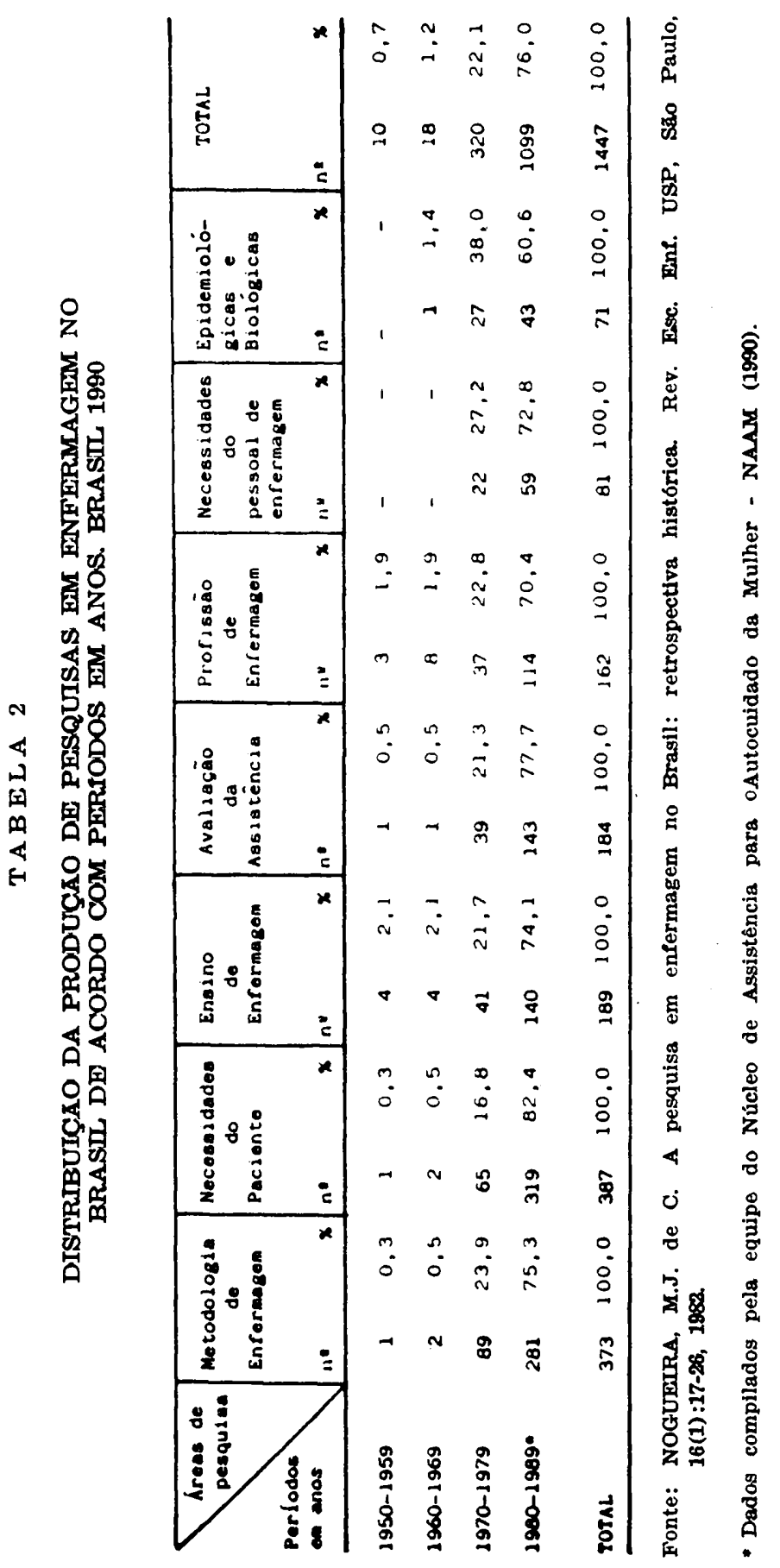


No Brasil, atualmente, os pesquisadores de enfermagem são principalmente enfermeiros docentes $4,5,19,22,24$.

A participação destes docentes $\mathrm{cm}$ realizações e publicações de pesquisas na enfermagem vem demonstrando um contínuo crescimento.

Alguns explicam esta evolução relacionando-a ao regime de dedicação exclusiva nas Universidades, implantado a partir de 1970 e que tem como requisito de admissão a apresentação de projeto de pesquisa e apresentação de relatórios subseqüentes ${ }^{19}$. Acreditamos que este possa ser considerado um fator de estímulo, porém não pode ser considerado como único.

Um outro fator que pode explicar este predomínio é o fato de que os docentes são os que mais freqüentemente são convidados para a apresentação de Temas Oficiais nos Congressos e Reuniões Científicas ${ }^{25}$.

A distância mantida pelos enfermeiros assistenciais da pesquisa, constitui um paradoxo para aqueles que estudam a pesquisa articulada à prática na busca de novas formas de fazer e conhecer a essência da enfermagem.

Alguns fatores parecem interferir nesta mobilização dos enfermeiros assistenciais em direção à produção do saber: o curso de graduação não tem valorizado suficientemente o preparo do aluno para a investigação; os serviços nem sempre oferecem oportunidade de educação continuada; os valores relacionados ao poder de decisão e comando sobrepujam aqueles relacionados à investigação; o desvio das funções, afastando o enfermeiro do objeto de sua ação ${ }^{25}$.

Apesar de incipiente, a inserção dos enfermeiros assistenciais no campo da pesquisa, já se fez notar por meio das tentativas de desenvolvimento de trabalho em conjunto ${ }^{22}$ e pelo crescente número de enfermeiros assistenciais admitidos nos cursos de Pós-Graduação no Pais.

\section{Procedência}

A localização temporal da produção científica é importante para a percepção das tendências desta produção, mas a localização espacial, desta produção, determinada pela procedência dos autores, define as regiões de excelência e de emergências do saber da enfermagem brasileira.

Assim é que em diversos estudos realizados nota-se uma caracterização significativa da região sudeste, origem de $63,8 \%$ dos autores em periódicos e 68,1 $\%$ dos autores de teses e monografias ${ }^{(25)}$.

A produção científica em enfermagem pediátrica também obedece esta seqüência correspondendo, $85,3 \%$ dos autores, para a região sudeste e $13,1 \%$ para a região sul seguida da região nordeste $1,6 \%{ }^{5}$.

No levantamento realizado em 1983 pelo Conselho Federal de Enfermagem (COFEn) e pela Associaçāo Brasileira de Enfermagem 
(ABEn), o Estado de São Paulo aparece, também, como maior produtor quantitativo de pesquisas realizadas por enfermeiros no País.

\section{Tipos de Pesquisa}

Num estudo realizado por Mendes \& Trevisan (1983) onde analisam teses e dissertações brasileiras apresentadas no período de 19631979 , buscando verificar a utilização do método científico, definem o método indutivo $(89,5 \%)$ como o mais utilizado pelos pesquisadores neste período. Os autores localizam esses trabalhos na fase de observação e experimentação, e extrapolam esses achados para os trabalhos publicados em periódicos. O método dedutivo compreende apenas $10,4 \mathrm{c} \%$ do total de trabalhos realizados pelos pesquisadores neste período.

Outros autores identificaram uma influência marcante do positivismo nas 285 dissertações e teses produzidas no Brasil no periodo de 1979 a 1984, e uma tendência emergente de propostas alternativas fundamentadas na dialética e na fenomenologia ${ }^{20}$.

Nesta década acontece o primeiro Evento Internacional sobre Pesquisa Qualitativa em Enfermagem, patrocinado pela Escola de Enfermagem da Universidade de São Paulo e o Departamento de Enfermagem da Universidade Federal de Santa Catarina ${ }^{24}$.

\section{A Aplicação de Pesquisas na Prática}

A aplicação prática das pesquisas realizadas no Brasil, só pode acontecer se ocorrer sua divulgação, aqui entendida como a comunicação ao público em geral ou à comunidade científica em particular, dos conhecimentos gerados pelo pesquisador.

No Brasil esta divulgação tem se processado de forma lenta porém evolutiva, e hoje podemos afirmar que há uma tendência para maior publicação de estudos e relatórios de pesquisa de enfermagem.

Temos hoje no Brasil sete revistas de enfermagem, destacando-se duas, pela extensão do período de circulação: a Revista Brasileira de Enfermagem (ABEn) e a Revista da Escola de Enfermagem da USP (EEUSP).

Algumas Universidades brasileiras mantêm um espaço para a enfermagem publicar seus artigos. Assim destacam-se: a Revista da Sociedade Brasileira para o Progresso da Ciência e a Revista Paulista de Hospitais, entre outras.

A divulgação dos resultados de pesquisas tem sido efetivada por meio de apresentação oral em eventos, congressos, seminários e escrita em boletins e jornais.

A inexistência de programas de integração docente-assistencial bem sucedidos, também contribui para o distanciamento e acentuada dicotomia prática-pesquisa e prática-ensino. 
Existem evidências de que o enfermeiro de serviço tem tido pouca participação em trabalhos de pesquisa, não aplicando seus resultados e até desconhecendo-os $\mathbf{2 , 7}$.

No entanto, há um clima de expectativa por parte dos enfermeiros hospitalares no que diz respeito à atuação como "parceiros em todas as fases do processo de pesquisa", visto como condição necessária para a incorporação dos resultados à prática assistencial 18,23.

Se a produção brasileira de pesquisas em enfermagem está concentrada nos docentes e se os enfermeiros assistenciais pouco conhecem sobre os resultados destas pesquisas e se não se conhecem os projetos bem sucedidos da integração docente-assistencial, parece-nos que aí está o ponto vulnerável do desenvolvimento integrado do saber e do fazer na enfermagem.

Teoria e pesquisa, neste contexto, vêm da prática e são melhoradas na prática, reforçando a articulação e integração dessa tríade.

Estudos relacionados ao tema demonstram que os enfermeiros assistenciais consideram como obstáculos à utilização dos resultados da pesquisa na prática: falha na divulgação dos resultados das pesquisas, difícil acesso às publicações, falta de tempo, recursos humanos e materiais para a operacionalização dos resultados ${ }^{11}$.

Assim, percebe-se que os enfermeiros brasileiros, à semelhança de outros países do primeiro, segundo e terceiro mundo, ainda mantêm um trabalho individualizado e não cooperativo no desenvolvimento de grande parte da ciência, da tecnologia e da assistência à saúde de sua clientela.

\section{PROGRAMAS EDUCACIONAIS EM NIVEL DE PÓS-GRADUAÇĀO - "STRICTU SENSU"}

A primeira escola de enfermagem brasileira que marcou a inserção da enfermagem na pós-graduação "strictu sensu", foi a Escola de Enfermagem Ana Neri em 1972. Não contando com professores de enfermagem com experiência em pesquisa, buscou-os em outras unidades da própria Universidade e em outras instituições.

A seguir, a Escola de Enfermagem da Universidade de São Paulo, 1973, e a Escola de Enfermagem de Ribeirão Preto, também da Universidade de São Paulo, em 1974, iniciaram seus cursos de pós-graduação.

Outras escolas se sucederam na criação dos cursos de Mestrado: Escola de Enfermagem da Universidade Federal da Bahia (1975), Universidade Federal de Santa Catarina (1976), Departamento de Enfermagem da Escola Paulista de Medicina (1978), Escola de Enfermagem da Universidade Federal da Paraíba (1979) e Escola de Enfermagem da UNI-Rio (1982).

Em 1980, a Escola de Enfermagem da Universidade de São Paulo e a Escola de Enfermagem de Ribeirão Preto, conseguiram a criação 
do Programa Interunidades em nivel de Doutorado, na Universidade de São Paulo, o primeiro da América Latina. A seguir em 1986 foi criado um curso de pós-graduação em nível de doutorado, pelo Departamento de Enfermagem da Escola Paulista de Medicina; em 1988 foi criado um outro curso de pós-graduação em nível de doutorado na Escola de Enfermagem da Universidade de São Paulo; e em 1989 foi criado o curso de doutorado em Enfermagem na Escola de Enfermagem Ana Neri.

Hoje temos oito cursos de pós-graduação em Enfermagem com as respectivas áreas de concentração, localizados em sua maioria na região Sudeste, um na região Sul e dois na região Nordeste.

\section{CURSOS DE MESTRADO E DOUTORADO}

\section{Árcas de Concentração}

EEUSP Fundamentos de Enfermagem

Enfermagem Obstétrica

Enfermagem Pediátrica

Administração de Serviços de

Doutorado EEUSP

Enfermagem 1988

Enfermagem Psiquiátrica

Enfermagem em Saúcie Coletiva (em estudo)

Doutorado Interunidades EEUSP/

EERPUSP - 1981

EERPUSP Enfermagem Psiquiātrica

Enfermagem Fundamental

UFSC

EPM

Saúde Pública

Enfermagem Pediátrica e

Pediatria Social

Doutorado 1986

Enfermagem Obstétrica e

Obstetricia Social

Saúde do Adulto

EEAN

Saúde da Comunidade

Doutorado 1989

Metodologia e Assistência

de Enfermagem

Enfermagem Psiquiátrica

Enfermagem Fundamental

Enfermagem Médico-Cirúrgica

Enfermagem Materno-Infantil

(em estudo)

UNI RIO Enfermagem em Saúde Pública

Enfermagem na Saúde do Adulto

UFBa

Enfermagem Médico-Cirúrgica

UFPb

Enfermagem em Saúde Pública 


\section{O PAPEL DAS AGENCIAS FINANCIADORAS \\ DE PESQUISA NA AREA DA ENFERMAGEM}

Em 1951 foram criados no Brasil o Conselho Nacional de Pesquisa (CNPq) e a Coordenação de Aperfeiçoamento de Pessoal de Nível Superior (CAPES). O primeiro órgão visando o financiamento de pesquisas e o segundo o preparo de recursos humanos de nivel superior, ambos de relevada importância para a enfermagem.

A Financiadora de Estudos e Projetos (FINEP), criada em 1967, teve como objetivo fomentar estudos, pesquisas, programas e projetos capazes de favorecer o desenvolvimento científico e tecnológico do País, atendendo a Universidades, centros e instituições de pesquisa e empresas nacionais.

Em São Paulo, em 1962, foi criada a Fundação de Amparo à Pesquisa no Estado de São Paulo (FAPESP) objetivando o fortalecimento da pesquisa no Estado, que vem assessorando outros Estados na formação de órgãos semelhantes.

A área de enfermagem nestas instituições faz parte da grande área saúde, junto com a medicina, nutrição, odontologia e fisioterapia.

Segundo estudo realizado por Adami et al, os agentes financiadores mais conhecidos pelos enfermeiros que cursam pós-graduação, são: o CNPq $(32 \%)$ e a CAPES $(21,4 \%)$. Entre outros agentes financiadores foram citados: o Ministério da Saúde e da Previdência Social OPS/OMS, Fundação Kellog, laboratórios farmacêuticos e bancos como - BID, Banco do Brasil e Banco do Nordeste.

Ainda neste estudo, os autores referem que 149 (quase $50 \%$ ) de enfermeiros pós-graduandos não solicitaram nenhum tipo de recurso aos órgãos financiadores, e dos que solicitaram, 64,7\% obtiveram os recursos solicitados ${ }^{1}$.

Podemos concluir que: as instituições de ensino de pós-graduação em enfermagem, as associações e outros órgãos devem procurar divulgar mais, as fontes de recursos para realização de pesquisas, no âmbito de sua atuação e que a demanda por estes recursos deve ser estimulada, visto que esta se reflete no crescimento àe recursos destinados para uma determinada área.

Um outro fator importante para o desenvolvimento de pesquisas em enfermagem é a preocupação de algumas Universidades do País, como por exemplo a Universidade de São Paulo, que criando um Conselho de Pesquisa, onde todes as suas Unidades têm representantes, exige um plano diretor de cada Unidade, no sentido de estabelecer um planejamento a médio e longo prazo para obtenção de recursos.

\section{PRIORIDADES DA PESQUISA EM ENFERMAGEM}

As prioridades oficiais para o desenvolvimento de pesquisas em enfermagem foram delineadas em 1982, por ocasião da realização do II Seminário Nacional sobre Ensino de Pós-Graduação e Pesquisa em 
Enfermagem, quando foram estabelecidas as linhas prioritárias de pesquisa em três grandes áreas: profissional, assistencial e estrutura, organização e funcionamento das instituições. (Anexo I)

As prioridades de pesquisa em enfermagem podem ser apresentadas sob dois enfoques: um geral, onde são analisados os fatores que influenciam de um modo abrangente a pesquisa na área de enfermagem, no âmbito nacional, regional e local, e o outro específico, relacionado à natureza própria da prática da enfermagem.

No primeiro enfoque destacam-se as diretrizes políticas emanadas de órgãos superiores e que caracterizam uma linha diretiva no sentido de preservar o equilíbrio entre a pesquisa e a tecnologia, como estágios de um processo articulado com a economia e comunidade.

As ações de saúde materno-infantil, o controle das doenças transmissiveis e as medidas de saneamento ambiental, são consideradas relevantes para a comunidade brasileira, envolvendo alocação de recursos específicos e desenvolvimento de programas especiais, em nível do $\mathrm{Mi}$ nistério da Saúde.

Já neste nivel, podemos ressaltar a necessidade do envolvimento das enfermeiras, no que diz respeito aos estudos que deveriam acompanhar a implementação dessas ações de saúde, dirigidas para as ações específicas de enfermagem, em sua caracterização de independência e dependência de outros profissionais.

Os resultados obtidos por meio de pesquisas operacionais permitiriam uma melhor adequação dos programas às realidades locais ou regionais; o estabelecimento de padrões mínimos de assistência de enfermagem, de supervisão, de controle e de avaliação, de forma a permitir o processo contínuo da coordenação; a compatibilização das ações de enfermagem destinadas a áreas específicas em nível federal, estadual e municipal; a disciplinação da aplicação de recursos alocados; a criação de um sistema eficiente de informação e a intercomunicação entre as áreas educaçāo, assistência e comunidade.

Há que se situar nesse contexto, a problemática atual da assistência à saúde, ou seja, o hiato existente entre a assistência curativa e a preventiva. Essa problemática reflete-se na enfermagem pela própria contingência da utilização quantitativa dos recursos humanos disponiveis, e envolve de tal modo o sistema formador, que se desenvolve um vínculo vicioso, cerceando o enfoque preventivo, mesmo quando elaborado sobre o curativo.

Consideramos prioritário, o desenvolvimento de estucios no sentido de posicionar a enfermeira como agente de promoção e proteção da saúde do cliente, família e comunidade, delineando o âmbito de ação da enfermeira em sua independência.

Na área da educação, o esboço de uma política de educação superior, refere-se, em suas diretrizes, a projetos prioritários a serem desenvolvidos a médio prazo. Destacam-se os novos planos curriculares, as novas metodologias de ensino, aprendizagem e de avaliação do ensino 
a nivel de graduação, bem como a integração do ensino superior na comunidade.

A saúde ocupacional, regulamentada pelo Ministério do Trabalho através da Portaria $\mathrm{n}^{\circ}$ 3.237, de 27 de julho de 1972, a partir de $1^{\circ}$ de janeiro de 1975, apresenta-se também como um contingente prioritário de estudos, dirigidos essencialmente para a promoção e proteção da saúde no ambiente de trabalho, envolvendo os riscos provenientes de condições insatisfatórias de segurança causadas por agentes físicos, químicos e biológicos.

Ainda no contexto do enfoque geral, as condições de desenvolvimento sócio-econômico da região, que envolvem o homem e o seu bem estar, em seu mais amplo sentido, são fatores intervenientes na determinação das prioridades da pesquisa. Espera-se que os estudos realizados nessa área contribuam para um conhecimento mais aprofundado e mais minucioso da realidade nacional, permitindo uma eficiência maior ao trabalho de todos os profissionais que, pelo caráter de suas atividades, são agentes da mudança social e cultural, incluindo-se nesse contexto as enfermeiras, com seus próprios recursos operacionais.

A extensão de cobertura dos serviços de saúde no Brasil, por meio de novas práticas, que vêm sendo implantadas, dinamizadas, racionalizadas, e/ou desconcentradas, fundamentam-se nas necessidades sentidas e recursos existentes no espaço urbano e rural.

Estudos referentes a utilização dos sistemas formal e informal de saúde envolvendo conceitos, conhecimentos, práticas e agentes informais utilizados, seriam de extrema valia às perspectivas atuais de extensão de cobertura.

O enfoque específico, relacionado à natureza própria da prática de enfermagem, visa a busca de conhecimentos necessários à reformulação de conceitos, à atualização de procedimentos fundamentados em princípios científicos, à validação das teorias de enfermagem, à solução dos problemas do paciente, família e comunidade, pertinentes à enfermagem, à elaboração de programas, à validação de critérios e padrões de assistência e ensino, constituindo-se em prioridades de acordo com as linhas de pesquisa adotadas nas instituições.

\section{LIMITAÇŌES}

É reconhecido o fato de que no Brasil há insuficiência de pesquisas no campo de enfermagem, que contribuam para a resolução de problemas específicos e para a determinação de políticas ajustadas ao desenvolvimento tecnológico e científico das ciências da saúde.

Dentre os fatores que poderiamos considerar como limitantes das atividades de pesquisa na enfermagem, ressaltamos o preparo do pesquisador.

Na nossa opinião, a motivação para esse preparo deveria emergir a partir da graduação, sendo desenvolvida ao longo da sua formação, continuadamente. 
O limite crítico, elaborado pela enfermeira em torno de sua própria criatividade, é por nós considerado como um outro fator relevante, por sua natureza ampla em extensão e profundidade. Percebemos ainda o comportamento estereotipado, executado rotineiramente ao longo de anos de prática compulsiva.

O custo elevado de qualquer investigação científica, por mais simples que ela seja, é um fator que limita a quantidade e a qualidade do produto da pesquisa. Assim, ao verificar o custo de exame bacteriológico, a enfermeira, por mais motivada que esteja, desistirá de sua idéia inicial e tentará desenvolver um estudo de menor custo operacional.

A sub utilização dos recursos da comunidade científica e dos órgãos financiadores é uma limitação para a investigação. Isto ocorre pela falta de divulgação na área da enfermagem, dos recursos disponiveis para a pesquisa e da falta de persistencia da enfermeira em tratar de obtê-los junto aos canais competentes.

O acesso aos sistemas de referência também pode ser consolidado como fator determinante de limitações da pesquisa.

A inexistência de laboratórios de pesquisa próprios para a enfermagem, com recursos suficientes e a deficiência de orientadores em termos de quantidade, qualidade e disponibilidade, são fatores relevantes na limitação da pesquisa.

A dicotomia do relacionamento docente assistencial, nega a sua interdependência como um conjunto, no qual, a mudança em uma das partes provoca um impacto sobre a outra atividando-a. Esta desarticulação é uma limitação para utilização do campo para pesquisa. Há que se observar, ainda, que em nosso meio, as pesquisas têm se desenvolvido sob forte pressão de tempo, título e/ou contrato, quando deveriam originar-se de problemas sentidos ou percebidos, e que impedem a equipe de enfermeiros de desenvolver um trabalho mais qualificado.

\section{PAPEL DA ASSOCIAÇĀO BRASILEIRA DE ENFERMAGEM NO DESENVOLVIMENTO DE PESQUISAS}

A primeira inserção da Associação Brasileira de Enfermagem no campo da pesquisa aconteceu em 1964, quando o Tema oficial do XVI Congresso Brasileiro de Enfermagem foi "Enfermagem a Pesquisa", e que teve como resultado as recomendações de estímulo às escolas de enfermagem no sentido de prepararem seus docentes para a pesquisa e utilização da pesquisa na prática ${ }^{3}$.

Em 1971, durante a realização do XXIII Congresso Brasileiro de Enfermagem, foi criado o Centro de Estudos e Pesquisas na Enfermagem (CEPEn) com o objetivo de coletar dados, estudá-los e divulgar os resultados de pesquisas. Este iniciou suas atividades em 1978.

Em 1979 é realizado o primeiro Seminário Nacional de Pesquisa em Enfermagem na cidade de Ribeirão Preto sob o patrocínio do $\mathrm{CNPq}$, 
a participação de enfermeiros neste evento foi limitada. Segundo a Comissão Executiva do evento, este seminário não apresentou "conclusões definitivas", entretanto permitiu reflexões sobre os temas apresentados e abriu novas perspectivas para a pesquisa em Enfermagem.

O segundo evento realizado pela $A B E n$ foi denominado: "Avaliação e Perspectivas do Ensino de Pós-Graduação e Pesquisa em Enfermagem", realizado em 1982 com cerca de 30 participantes, na cidade de Brasília e definiu as linhas prioritárias da pesquisa na área da enfermagem. (Anexo I)

O terceiro Seminário Nacional de Pesquisa, aconteceu em 1984 na cidade de Florianópolis - S.C., contou com participação não limitada e visou principalmente as abordagens metodológicas, caracterizando a evolução do interesse em pesquisas por parte dos enfermeiros.

O quarto Seminário Nacional de Pesquisa em Enfermagem foi realizado em 1985 na cidade de São Paulo, com o objetivo de estudar a produção de pesquisas em enfermagem no Brasil e suas tendências.

Em 1988 aconteceu o quinto Seminário Nacional de Pesquisa em Enfermagem, em Belo Horizonte - M.G., cujos Anais ainda não foram publicados.

A ABEn, por meio do CEPEn, iniciou em 1979 a publicação do Informações sobre Pesquisas a Pesquisadores em Enfermagem, que se encontra atualmente no VIII volume - 1989.

\section{CONCLUSOES}

A evolução quantitativa e qualitativa das pesquisas em enfermagem no Brasil, associada ao perfil dos pesquisadores e à implementação de programas de pós-graduação, nos permitem afirmar que neste ritmo a produção do saber específico de enfermagem, acompanhada de uma estrutura operacional de suporte e do envolvimento de enfermeiros assistenciais, garantirá a territorialidade da enfermagem brasileira no estudo e na resolução dos problemas de saúde do País.

CIANCIARULLO, T.I.; SALZANO, S.D.T. The nursing and research in Brazil. Rev. Esc. Enf. USP, v. 25, n. 2, p. 195-215, Aug. 1991.

The brazilian nursing in relation to research presented an important development in the last few years, with a quantitative improvement in the last decade.

The brazilian researchers are concentrated in its majority among the faculty members and in the Southeast of Brazil.

There are eight graduate university centers with several knowledge areas of concentration at master level. The doctoral level programs are all in the Southeast.

There are resources available in research financial agencies for nursing, however the demand is still incipient.

The Brazilian Nursing Association has tried to implement procedures which aim the development of researchers in nursing.

U.NITERMS: Nursing research. Nursing - Brazil. 


\section{REFERENCIAS BIBLIOGRAFICAS}

1. ADAMI, M.P.; RODRIGUES, M.A.; MARTINS, C.L.; RIGAUD, H.M.; SHOR, N. Utilização de agências financiadonas para pesquisa: a ótica de coordenadores de cursog e de alunos de pós-graduacão em enfermagem e em saúde pública. In: SBMINARIO NACIONAL DE PESQUiSA EM ENFERMAGEM, 4, São Paulo, 1985. Anais. São Paulo, Associação Brasileira de Enfermagem, 1985. p. 162-92.

2. ALMEIDA, M.H. O conhecimento dos enfermeiros sobre os resultados de pesquisa em enfermagem. In: SEMINARIO NACIONAL DE PESQUISA EM ENFERMAGEM, 4, São Paulo, 1985. Anais. São Paulo, Associação Brasileira de Enfermagem, 1985. p. 243-59.

3. ANGerani, E.L.S. Prioridade de investigação em enfermagem, Rev. Prul. Fnnt., v. 5, n. 2, p. $47-53,1985$.

4. BIANCHI, E.R.F.; PENICHE, A.C.G.; GATTO, M.A.F.; SILVA, A.; SALZANO, S.D.T. Estudo retrospectivo de pesquisa de enfermagem em centro cirúrgico. Enfoque, v. 16, n. 3, p. $75-7,1988$.

5. BONILHA, A.L.L.; HORTA, A.L.M.; RIBEIRO, M.O. Pesquísa em enfermagem pediatrica. Rev. Esc. Enf. USP, v. 21, n. 2, p. 117-34, 1987.

6. CASSIANI, S.H. de B. A coleta de dados nas pesquisas de enfermagem: estratégias, validade e confiabilidade. Ribeirão Preto, 1987. Dissertação (mestrado) - Escola de Enfermagem de Ribeirão Preto, Universidade de Săo Paulo.

7. CASTRO, I.B.; MIRANDA, C.M.L.; RODRIGUES, A.P.S.; LIMA, M.Y. Dificuldades na incorporação dos resultados de pesquisa na prática de enfermagem. In: SEMINARIO NACIONAL DE PESQUISA EM ENFERMAGEM, 4, São Paulo, 1985. Anais. São Paulo, Associação Brasileira de Enfermagem, 1985. p. 193-242.

8. CONSELHO FEDERAL DE ENFERMAGEM. O exercício da enfermagem nas instituiçōes de saúde do Brasil 1982/1983: força do trabalho em enfermagem: Rio de Janeiro, ABEn, 1985. v. 1, cap. 10, n. 171-81: Pesquisa de produção cientifica do enfermeiro.

9. COZZUPOLI, C.A.; GARCIA, T.Y.M. Pesquisa em enfermagem materno infantil. In: SEMINARIO NACIONAL DE PESQUISA EM ENFERMAGEM, 4, São Paulo, 1985. Anais. São Paulo, Associação Brasileira de Enfermagem, 1985. p. 31-41.

10. KOIZUMI, M.S.; TAKAHASHI, E.I.U.; MIYADAHIRA, A.M.K. Pesquisa em enfermagem médico-cirúrgica. In: SEMINARIO NACIONAL DE PESQUISA EM ENFFRMAGEM, 4, São Paulo, 1985. Anais. São Paulo, Associação Brasileira de Enfermagem, 1985. p. 61-77.

11. MASSAROLLO, M.C.K.B.; CUNHA, K. de C.; CIAMPONE, M.H.T.; BRAZ, H.; GAGLIARDI, E.M.D.B.; BEVILACQUA, D.F.; MAFDA, S.T.; SILVA, M.A.C.; MARKIEVICZ, Y. Utilização da pesquisa de enfermagem na prática. Rov. Paul. Ent., v. 6, n. 4, p. 146-56, 1986.

12. MENDES, I.A.C.; TREVISAN, M.A. Acerca da utillzação do método clentifico nas pesquisas de enfermagem. Kov. Bras. Enf., v. 36, n. 1, p. 13-9, 1983.

13. Miranda, L.C.M. País precisa dos centros de pesquisa. Folha de Sa Paulo, Săo Paulo, 18-mar.-1987. Cad. 1.

14. NEIRA HUERTA, E del P.; MADEIRA, L.M.; MIRANDA, N.M.; MERIGHI. M.A.B.: HO, S.F.S.; GARCIA, T.R. A pesquisa em enfermagem materno infantil. Rov. Eac. Fint. USP, v. 17, n. 3, p. 213-27, 1983. 
15. NOGUEIRA, M.J. de C. A pesquisa em enfermagem no Brasil: retroepectiva histórica. Rev. Esc. Ent. USP, v. 16, n. 1, p. 17-26, 1982.

16. NOGUEIRA, M.J. de C. Pesquisa em enfermagem comunitária. In: SFMINARIO NACIONal DE PESqUiSA EM enfermagem, 4, São Paulo, 1985. Anais. Săo Paulo, Assoclaçăo Brasileira de Enfermagem, 1985. p. 45-59.

17. PADILHA, K.G.; PIERINT, A.M.G.; IDE, C.A.C.; BRAZ, E.; FARIAS, G.M.; TAK HASHI, O.C. Pesquisa em enfermagem médico-cirúrgica no Brasil. Rev. Paul. Inf., v. 3, n. 5, p. $167-9,1983$.

18. RIBEIRO, C.M. A pesquisa $\theta$ a prática de enfermagem. In: SEMINARIO NACIONAL DF PHSQUISA EM ENFERMAGFM, 3, Florianópolis, 1984. Anais. Florianópolis, Editora da Universidade Federal de Santa Catarina, 1984. p. 10-26.

19. ROCHA, M.L.Q. Reflexões sobre a posição dos pesquisadores e da comunidade irente aos resultados da pesquia de enfermagem. In: SEMINARIO NACIONAL DB PBSQUISA EM ENFtgRMAGEM, 4, São Paulo, 1985. Anais. São Paulo, Asgociaça Brasileira de Entermagem, 1985. p. 143-51.

20. ROCHA, S.M.M.; SILVA, G.B. Linhas filos6ficas e ideológicas na pesquisa em enfermagem no Brasil. Rev. Bras. Ent., v. 40, n. 4, p. 214-21, 1987.

21. RUF'iNo, M.C.; FREITAS, D.M.V. de; CASAGRANDE, L.D.R. Retrospectiva das publicacões de pesquisa realizadas sobre educação em enfermagem: 1947-1981. Rev. Bras. Int., v. 38, n. 3/4, p. 245-56, 1985.

29. SPAGNOLO, F.; GUNThR, H. 20 anos de pós-graduação: o que fazem nossos mestres e docentes? Brasilia, CAPES, 1985.

23. STFFANELLI, M.C.; FUKUDA, I.M.K.; ROLIM, M.A.; ARANTES, E.C. Situacåo da pesquisa em enfermagem psiquíatrica no Brasil. Rev. Bras. Enf., v. 40, n. 1, p. 60-5, 1987.

24. STEFANELII, M.C.; SAIZANO, S.D.T.; OGUISSO, T. Sítuação da pesquisa qualltativa em enfermagem no Brasil. Rev. Paul. Ent., v. 9, n. 2, p. 50-6, 1990.

25. TRENTINI, M. Relacăo entre teoria, pesquisa e prática. Rev. Ese. Enf. USP, v. 21, n. 2, p. 135-43, 1987.

26. VIFIRA, T.T. Produçåo clentifica em enfermagem no Brasil: 1970-1979. Salvador, 1980. Tese (Professor Titular) - Escola de Enfermagem, Universidade Federad da Bahia 


\section{A N EXO I}

\section{PRIORIDADES DE PESQUISA EM ENFERMAGEM}

\section{Profissional}

Linha 1 - A Enfermagem como Prática Social:

estudos sobre objeto de trabalho da Enfermagem; estudos sobre as relações do enfermeiro com sua clientela; estudos sobre o espaço que o enfermeiro ocupa no mercado de trabalho; estudos dos fatores culturais, históricos, políticos, sociais, econômicos determinantes no desenvolvimento profissional; estudos sobre a autonomia profissional; estudos sobre relações internas e externas do trabalho de Enfermagem.

\section{Linha 2 - Formação e Utilização dos Recursos Humanos em Enfermagem:}

estudos sobre o currículo de Enfermagem; estudo sobre a adequação do enfermeiro à prática profissional; estudo sobre a integração docente-assistencial; estudo sobre a metodologia do ensino-aprendizagem e avaliação do processo ensino-aprendizagem; estudos sobre os fatores históricos, culturais e sociais determinantes do processo ensino-aprendizagem; estudos sobre a relação mercado de trabalho e a profissâo de Enfermagem; estudos sobre a divisão social do trabalho; estudos sobre a força de trabalho e dimensionamento de recursos humanos (qualitativos e quantitativos).

\section{Assistencial}

Linha 1 - Fundamentação da Assistência, Tecnologia e Instrumentação:

estudos sobre a renovação de procedimentos técnicos; estudos sobre desenvolvimento e testagem das teorias e conceitos de enfermagem; estudos sobre a adequação de recursos tecnologicos.

Linha 2 - Autoćuidado à Saúde:

estudos sobre a busca de respostas a perguntas sobre condições de ajuda às pessoas; estudos da difusão do conhecimento sobre a saúde.

Linha 3 - Riscos da Assistência de Enfermagem:

estudos sobre a prática da assistência de enfermagem em termos de danos ou prejuizos causados à clientela.

\section{Linha 4 - Determinantes do Processo Saúde-Doença:}

estudos sobre o estilo de vida e saúde das populações; estudos sobre as necessidades e problemas da clientela; estudos sobre crenças, atitudes, comportamentos e necessidades em saúde; estudos epidemiológicos de morbidade e mortalidade. 
Estrutura, Organização e Funcionamento das Instituiçðes

Linha 1 - Modelos de Assistencia de Enfermagem:

estudos sobre a criação, implantação de modelos de assistência; estudos sobre o custa/eficiência da utilização desses modelos.

Linha 2 - Oferta, Acessibilidade e Utilização de Serviços de Saúde:

estudos sobre a oferta/demanda dos serviços de Saúde; estudos sobre a distribuição, acessibilidade, expansão de cobertura e utilização dos serviços de Saúde.

Linha 3 - Sistema de Informação em Enfermagem:

estudos sobre a informática; estudos sobre o sistema de comunicação; estudos sobre o sistema de divulgação e documentação de Enfermagem.

Linha 4 - Incorporação de Conhecimentos à Prática Profissional:

estudos sobre a produção e a utilização do conhecimento de Enfermagem; estudos sobre a utilização de conhecimentos produzidos na melhoria da prática profissional.

Linha 5 - Dinamica das Organizaç̃es:

estudo das organizaçoes em movimento; estudos sobre conflitos; estudos sobre lideranças; estudos sobre o trabalho em equipe multiprofissional.

Fonte: BRASIL, SEPLAN, CNPq - Avaliagåo \& Perspectivas, Braslila, 1982, p. 176-177 (Ciencias da Saúde, v. 6, Enfermagem 38).

Recebido em 6-8-91

Aprovado em 20-6-91 